\title{
The power of some nonparametric test statistics for the location and mean slippage problems
}

\author{
Ru-Rong Hsiao* and Charles E. McCulloch \\ Biometrics Unit, Cornell University, Ithaca, NY 14853, USA \\ Ruey-Beei Wu \\ Department of Electrical Engineering, National Taiwan University, Taipei, Taiwan, ROC
}

Received 13 October 1989; revised manuscript received 1 November 1990

Recommended by T.J. Santner

\begin{abstract}
Many nonparametric test statistics for the location problem or mean slippage problem are based on extreme order statistics. A generalized class of statistics related to those of Rosenbaum (1954) and Mosteller (1948) are considered in this paper. Analytic formulae for the large sample power of these test statistics are derived for Lehmann alternatives and for pure-shift alternatives for various common population distributions. Under Lehmann alternatives, the large sample power is independent of the population distributions and the limiting power is less than one, which implies that these test statistics are asymptotically poor. The large sample power under pure-shift alternatives depends greatly on the population distributions. For example, it tends asymptotically to one for uniform or normal distributions but converges to the significance level for lognormal or Cauchy distributions. A simulation study indicates that the large sample results are highly predictive of the small and moderate sample size behavior. Care must therefore be exercised in the use of such tests for long-tailed distributions.
\end{abstract}

AMS Subject Classification: Primary 62G10; secondary 62G20.

Key words and phrases: Lehmann alternatives; order statistics; negative binomial distribution; asymptotic distribution; tail behavior.

\section{Introduction}

Many nonparametric test statistics for the location problem or the mean slippage problem are based on extreme order statistics. For example, Roscnbaum (1954) for the location problem and Mosteller (1948) for the mean slippage problem, considered the statistic equal to the number of observations from one population

\footnotetext{
* Current address: 2F, No. 5, Alley 12, Lane 310, Section 3, Muh Shin Rd., Muh Jah, Taipei, Taiwan, ROC.
} 
greater than the largest observation from the other populations. Related work on the mean slippage problem has been done by Bofinger $(1965)$, Conover $(1965,1968)$, Joshi and Sathe $(1978,1981)$, Neave $(1966,1972,1973,1975)$ and Neave and Granger (1968). Related work on the location problem has been done by Tukey (1959) and Rosenbaum (1965). In this paper we consider generalizations of the statistics of Mosteller and Rosenbaum. We show that the large sample power of the nonparametric tests considered are highly sensitive to the form of the alternative distribution. For distributions which are quite similar in practice, the large sample power can converge to one, to the significance level or to a constant in between. A simulation of Rosenbaum's statistic shows that the large sample results accurately predict the small and moderate sample size behavior. Thus care must be exercised before using these types of statistics with medium- or heavy-tailed distributions.

To investigate the power behaviors of these nonparantetic tests, the problem primarily considered in this paper consists of $k$ populations among which the $k$-th one is suspected to be stochastically larger than the first $k-1$ populations. An application of this type of test could be to an environmental monitoring situation, where independent samples are taken through time and a potential pollution source is introduced. In such a case we would be interested in testing if the time point after the introduction of the potential pollution source is stochastically larger than the rest. Ott (1984, p. 192) describes an example and illustrates the use of a test such as we consider for the two-sample situation. Section 2 proposes a new test procedure for which the distribution of the test statistic under the null hypothesis is derived and the critical region is determined. Then, two kinds of alternative hypotheses are considered to investigate their power behaviors, especially in cases of large sample sizes. In Section 3, we consider Lehmann alternatives. The distribution and power of the test statistics are derived and the effects on the large sample power behaviors due to different test statistics and alternatives are investigated. The limiting power against pure-shift alternatives is discussed in Section 4. Several common population distributions are included to depict the dependence of power behavior on the tails of the population distributions. Section 5 reports a Monte Carlo simulation which supports the results of Sections 3 and 4. In Section 6 we relate our problem to the slippage problem and finally, some discussions and conclusions are presented in Section 7.

\section{Test statistics for the location problem}

The setting considered here will be random samples of size $n_{i}$ from each of $k$ populations $(i=1,2, \ldots, k)$. For simplicity we will use $N=\sum_{i=1}^{k-1} n_{i}$ to denote the total sample size for the first $k-1$ populations and $n=n_{k}$ to denote the sample size for the population suspected to be to the right of the others. The first $k-1$ populations are identically distributed with cumulative distribution function (c.d.f.) $F(x)$ and the c.d.f. for the $k$-th population will be denoted $F_{k}(x)$. The null hypothesis of 
no slippage is then given by

$$
\mathrm{H}_{0}: F_{k}(x)=F(x)
$$

and the alternative hypothesis will be

$$
\mathrm{H}_{\mathrm{A}}: F_{k}(x)<F(x) \text { for all } x \text {. }
$$

Generalizing Mosteller's (Mosteller, 1948) and Rosenbaum's (Rosenbaum, 1954) test procedures, we consider test statistics $T_{r}$, which are defined by counting the number of observations in the $k$-th population which are greater than the $r$-th largest observation in the remaining $k-1$ populations. In other words, let $X_{i, j}$ denote the $j$-th observation from the $i$-th population and $Y_{N-r+1}$ be the $r$-th maximum in the observations $X_{i, j}\left(j=1,2, \ldots, n_{i} ; i-1,2, \ldots, k-1\right)$; then

$$
T_{r}=\sum_{j=1}^{n} S_{r, j}
$$

where $S_{r, j}$ is an indicator function defined as

$$
S_{r, j}= \begin{cases}1 & \text { if } X_{k, j}>Y_{N-r+1} \\ 0 & \text { if } X_{k, j} \leq Y_{N-r+1}\end{cases}
$$

For example, $T_{1}$ is the number of observations in the $k$-th population larger than the largest observation from the first $k-1$ populations.

The distribution of the test statistics can be determined from the order statistics $N_{N-r+1}$. Since all the $N$ observations from the first $k-1$ populations are identically and independently distributed with c.d.f. $F(x)$, we can make the mathematical simplification of reducing the problem to a two-sample problem where the sample size from $F(x)$ is $N$ and the sample size from $F_{k}(x)$ is $n$. The distribution of $Y_{N-r+1}$ is therefore given by Mood, Graybill, and Boes (1974),

$$
F_{Y_{N},+1}(y)=\sum_{h=0}^{r-1}\left(\begin{array}{l}
N \\
h
\end{array}\right)[F(y)]^{N-h}[1-F(y)]^{h},
$$

and the density function is

$$
f_{Y_{N}, 1}=r\left(\begin{array}{c}
N \\
r
\end{array}\right)[F(y)]^{N-r}[1-F(y)]^{r-1} f(y) .
$$

It is noted by inspecting (2.3) that the distribution of $T_{r}$ conditional on $Y_{N-r+1}$ is binomial with parameters $n$ and $p=1-F_{k}(y)$. Hence, the mass function of $T_{r}$ can be expressed by

$$
P\left(T_{r}=t\right)=\int_{-\infty}^{\infty} P\left(T_{r}=t \mid Y_{N-r+1}=y\right) f_{Y_{N} r+1}(y) \mathrm{d} y
$$




$$
\begin{aligned}
&=\int_{-\infty}^{\infty}\left(\begin{array}{c}
n \\
t
\end{array}\right) F_{k}(y)^{n-t}\left[1-F_{k}(y)\right]^{t} \\
& \cdot r\left(\begin{array}{c}
N \\
r
\end{array}\right)[F(y)]^{N-r}[1-F(y)]^{r-1} f(y) \mathrm{d} y
\end{aligned}
$$

for $t=0,1,2, \ldots, n$.

Under the null hypothesis (2.1), the mass function of $T_{r}$ can be found explicitly from (2.7), i.e.,

$$
P\left(T_{r}=t\right)=\frac{\left(\begin{array}{c}
t+r-1 \\
t
\end{array}\right) \cdot\left(\begin{array}{c}
n+N-r-t \\
n-t
\end{array}\right)}{\left(\begin{array}{c}
n+N \\
n
\end{array}\right)}, t=0,1,2, \ldots, n .
$$

For the large-sample case where $N \rightarrow \infty$ and $n / N \rightarrow \lambda,(2.8)$ can be simplified to

$$
P\left(T_{r}=t\right)=\left(\begin{array}{c}
t+r-1 \\
t
\end{array}\right)\left(\frac{\lambda}{1+\lambda}\right)^{t}\left(\frac{1}{1+\lambda}\right)^{r}, \quad t=0,1,2, \ldots
$$

This is a negative binomial distribution with parameters $r$ and $p=\lambda /(1+\lambda)$.

Intuitively, the statistical inference is to reject $\mathrm{H}_{0}$ if the test statistic $T_{r}$ is too large. Since $T_{r}$ is a discrete random variable, a randomized test must be employed to exactly achieve most prescribed significance levels $\alpha$. Therefore, to achieve an $\alpha$ level test we use the following procedure. Choose the integer $Z_{\mathrm{c}}$ and the probability $P_{c}$ such that

$$
\alpha=P\left(T_{r}>Z_{\mathrm{c}}\right)+P_{\mathrm{c}} P\left(T_{r}=Z_{\mathrm{c}}\right) .
$$

We then reject $\mathrm{H}_{0}$ if $T_{r}>Z_{\mathrm{c}}$ or, with probability $P_{\mathrm{c}}$, if $T_{r}=Z_{\mathrm{c}}$.

\section{Power against Lehmann alternatives}

Lehmann alternatives are mathematically natural nonparametric alternatives since, for them, the distribution of ranks is independent of the population distribution (Lehmann, 1953). We therefore derive the power of the test statistics $T_{r}$ against these alternatives, which are defined in terms of a parameter $m$ by

$$
\mathrm{H}_{\mathrm{A}}: F_{k}(x)=[F(x)]^{1 / m}, \quad 0<m<1 .
$$

Obviously, (3.2) satisfies the relation (2.2). Also, the mean of the $k$-th population moves to the right as $m$ gets smaller. However, the dependence of the variance on $m$ is complicated. For example, as $m$ gets smaller, the variance gets smaller for uniform or normal distributions; gets larger for lognormal or Cauchy distributions; and stays approximately the same for exponential distributions. These arguments will be made clear in Section 4 .

Equation (2.7) can be applied to derive the distribution of $T_{r}$. By a change of 


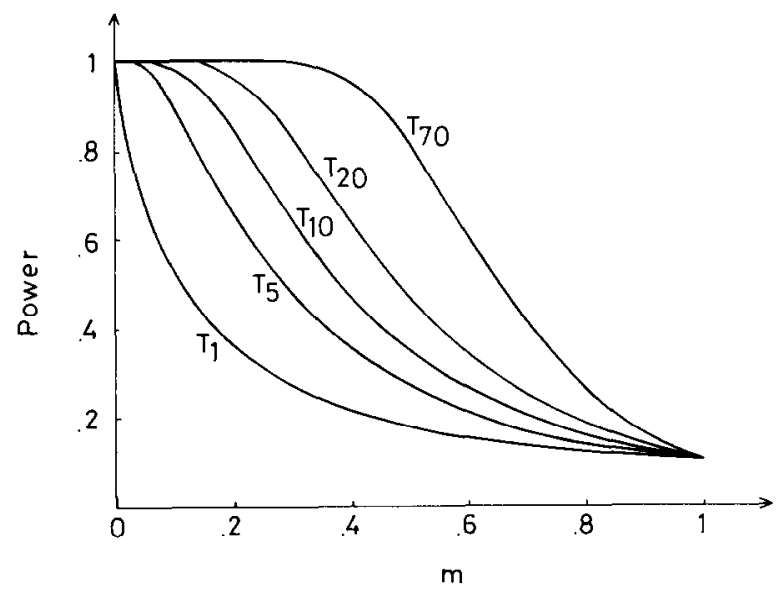

Fig. 1. Power versus $m$ while keeping $k=10, \alpha=0.1$ and $r=1,5,10,20$ or 70 .

variable $F_{k}(x)=g$, i.e., $F(y)=g^{m}$ and $f(y) \mathrm{d} y=m g^{m-1} \mathrm{~d} g$, as well as a binomial expansion for the term $[1-F(y)]^{r-1}=\left(1-g^{m}\right)^{r-1},(2.7)$ can be integrated to be

$$
\begin{gathered}
P\left(T_{r}=t\right)=N \cdot m \cdot\left(\begin{array}{c}
n \\
t
\end{array}\right)\left(\begin{array}{c}
N-1 \\
r-1
\end{array}\right) \sum_{h=0}^{r-1}(-1)^{h}\left(\begin{array}{c}
r-1 \\
h
\end{array}\right) \\
\cdot \mathrm{B}(t+1, n-t+m N-m r+m h+m),
\end{gathered}
$$

where $\mathrm{B}(\cdot, \cdot)$ denotes the Beta function.

Though (3.2) is valid for any finite sample size, the expression becomes much simpler in the limiting case. It is noted that the sequence of limit and integration in (2.7) can be interchanged according to the dominated convergence theorem (Folland, 1984). By defining a new variable $v=-n \cdot \log \left[F_{k}(y)\right]$, the limit of (2.7) as $N \rightarrow \infty$ and $n / M \rightarrow \lambda$ can be expressed in terms of $A=1+m / \lambda$ by

$$
\begin{aligned}
P\left(T_{r}=t\right) & =\frac{1}{t !} \cdot \frac{1}{(r-1) !} \cdot(A-1)^{r} \int_{0_{r}}^{\infty} v^{t+r-1} \mathrm{e}^{-A v} \mathrm{~d} v \\
& =\left(\begin{array}{c}
t+r-1 \\
t
\end{array}\right)\left(\frac{1}{A}\right)^{t}\left(1-\frac{1}{A}\right)^{r}, \quad t=0,1,2, \ldots .
\end{aligned}
$$

This is again a negative binomial distribution, but this time with parameters $r$ and $p=1 / A$ (compare (2.9)).

By employing (3.2) or (3.3), the power of $T_{r}$ against the Lehmann alternative can be calculated. Shown in Figure 1 is the large-sample power of test statistics $T_{r}$ with $r=1,5,10,20$ or 70 versus the parameter $m$ when the number of populations is $k=10$ and the significance level is $\alpha=0.1$. Obviously, the power of the test statistics $T_{1}$, is very poor even though the population size tends to infinity. It seems that a better way to improve the power is to increase the order of the test, $r$, instead of just the sample size $n$. 


\section{Power against pure-shift alternatives}

The most common alternative used in the mean slippage problems is the pure-shift alternative, which is defined as

$$
\mathrm{H}_{\mathrm{A}}: F_{k}(x)=F(x-\theta), \quad \theta>0 .
$$

The distribution of the $k$-th population here is uniformly shifted to the right of the first $k-1$ populations by a distance $\theta$. However, the distribution of ranks as well as the distribution of $T_{r}$ is now dependent not only on $\theta$ but also on the c.d.f. $F(x)$. It is thus very difficult to investigate the power under a pure-shift alternative analytically for finite sample-size cases. Therefore, only the limiting distribution of $T_{r}$, which is simpler but still illustrative, is emphasized here.

To find the limiting distribution of $T_{r}$, it is necessary to use the asymptotic distribution of order statistics. Let $Y_{n-j+1: n}$ be the $j$-th maximum in a random sample of size $n$ drawn from a given population. For most of the common population distributions, an asymptotic distribution for the extreme order statistic $Y_{n: n}$ exists (Galambos, 1978), i.e., there exist $a_{n}$ and $b_{n}$ such that

$$
\lim _{n \rightarrow \infty} P\left(\frac{Y_{n: n}-a_{n}}{b_{n}} \leq y\right)=G(y) .
$$

The c.d.f. $G(y)$ is the asymptotic distribution of the normalized extreme order statistic $Z_{1} \equiv\left(Y_{n: n}-a_{n}\right) / b_{n}$. Table 1 lists $a_{n}, b_{n}$, and $G(y)$ for some typical distributions (uniform, normal, exponential, lognormal, and Cauchy).

If the asymptotic distribution $G(y)$ for the normalized extreme order statistic $Z_{1}$ exists, the asymptotic distribution $G_{j}(y)$ for the normalized $j$-th maximum $Z_{j} \equiv$ $\left(Y_{n-j+1: n}-a_{n}\right) / b_{n}$ also exists and is related to $G(y)$ by (Galambos, 1978)

$$
\begin{aligned}
G_{j}(y) & =\lim _{n \rightarrow \infty} P\left(\frac{Y_{n-j+1: n}-a_{n}}{b_{n}} \leq y\right) \\
& =G(y) \sum_{h=0}^{j-1} \frac{1}{h !}\left[\log \frac{1}{G(y)}\right]^{h} .
\end{aligned}
$$

It is interesting to note that the distribution $F_{k}(x)$ under the Lehmann alternative (3.1) with $m=1 / n$ is identical to the distribution of the extreme order statistic $Y_{n: n}$. This implies that to discuss the change of distribution as the Lehmann shift $m$ tends to zero is no more than to discuss the behavior of extreme order statistics as $n$ tends to infinity.

Using (4.3), it is easy to derive the asymptotic distribution of test statistics $T_{\text {, }}$ under pure-shift alternatives. Obviously, the event $T_{r} \geq t$ means equivalently that the $r$-th maximum in the first $k-1$ populations, denoted $Y_{N-r+1: N}^{(\mathrm{I})}$, is smaller than the $t$-th maximum in the $k$-th population, denoted $Y_{n-t+1: n}^{(\text {II) }}$, i.e.,

$$
P\left(T_{r} \geq t\right)=\left(Y_{n-t+1: n}^{(\mathrm{II})}>Y_{N-r+1: N}^{(\mathrm{I})}\right)
$$




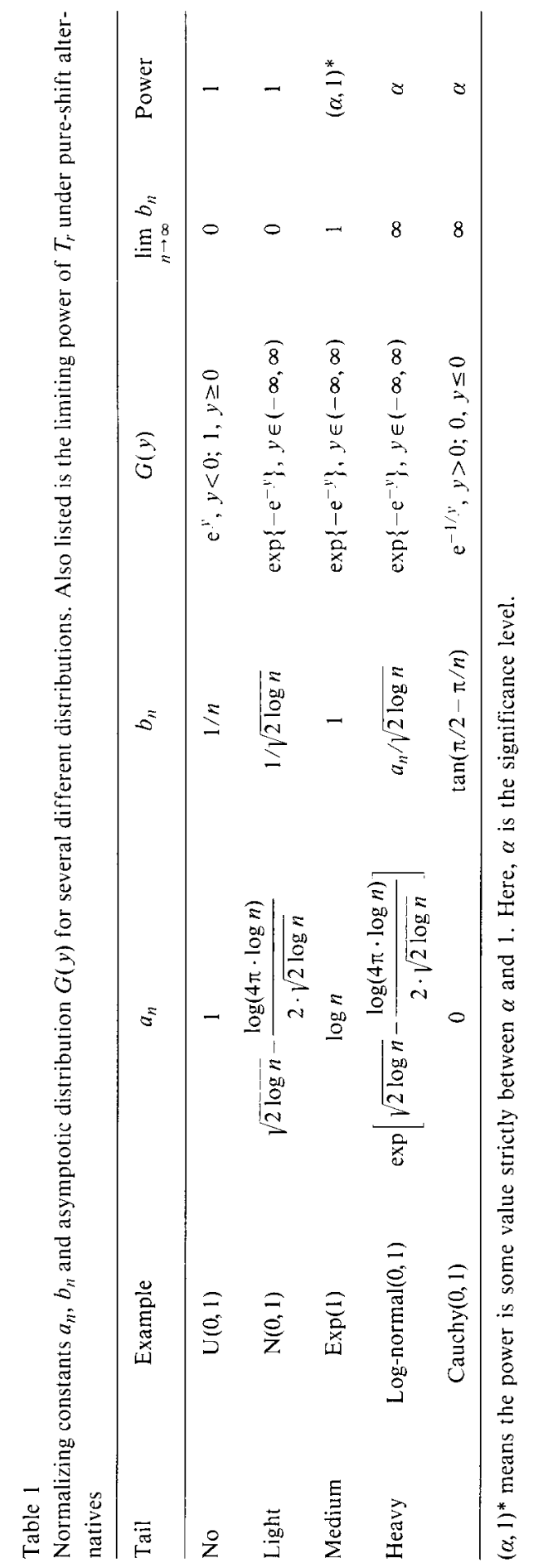


Suppose that the normalization constants $a_{n}, b_{n}$ and asymptotic distribution $G(y)$ for the distribution function $F(x)$ exist. Since the distribution of the $k$-th population, $F_{k}(x)$, is identical to $F(x)$ except for a shift by $\theta$, it is advantageous to define the following normalized order statistics

$$
Z_{r}^{(\mathrm{I})}=\frac{Y_{N-r+1: N}^{(\mathrm{I})}-a_{N}}{b_{N}}, \quad Z_{t}^{(\mathrm{II})}=\frac{\left(Y_{n-t+1: n}^{(\mathrm{II})}-\theta\right)-a_{n}}{b_{n}} .
$$

Then, $Z_{r}^{(\mathrm{I})}$ and $Z_{i}^{(\mathrm{II})}$, which are independent of each other, have asymptotic distributions $G_{r}(y)$ and $G_{t}(y)$, respectively.

Substituting (4.5) into (4.4), the asymptotic distribution of $T_{r}$ satisfies

$$
P\left(T_{r} \geq t\right)=\lim _{n \rightarrow \infty} P\left(Z_{t}^{(\mathrm{II})}-Z_{r}^{(\mathrm{I})} B_{k-1}>A_{k-1}-\theta / n_{n}\right)
$$

where

$$
A_{k-1}=\lim _{n \rightarrow \infty} \frac{a_{N}-a_{n}}{b_{n}}, \quad B_{k-1}=\lim _{n \rightarrow \infty} \frac{b_{N}}{b_{n}} .
$$

By (4.5), the mass function and thus the power of $T_{r}$ can be expressed in terms of the constants $A_{k-1}, B_{k-1}$ and $b_{n}$. Among these, the constant $b_{n}$ is crucial since it governs the large sample power. Typically, the power tends to one no matter how small $\theta$ is if $b_{n}$ tends to zero, while it tends to the significance level $\alpha$ no matter how large $\theta$ is if $b_{n}$ tends to infinity. If $b_{n}$ tends to a constant then inspection of (4.6) shows that the power tends to a constant in the interval $(\alpha, 1)$.

For distributions with finite upper range such as the uniform distributions, it can be shown that $b_{n}$ tends to zero as $n$ tends to infinity. Hence, the large sample power tends to one, i.e., the test statistics $T_{r}$ are asymptotically ideal. For distributions with infinite upper range, the situation becomes much more complicated since the constant $b_{n}$ may tend to zero, a nonzero constant, or infinity, as depicted in

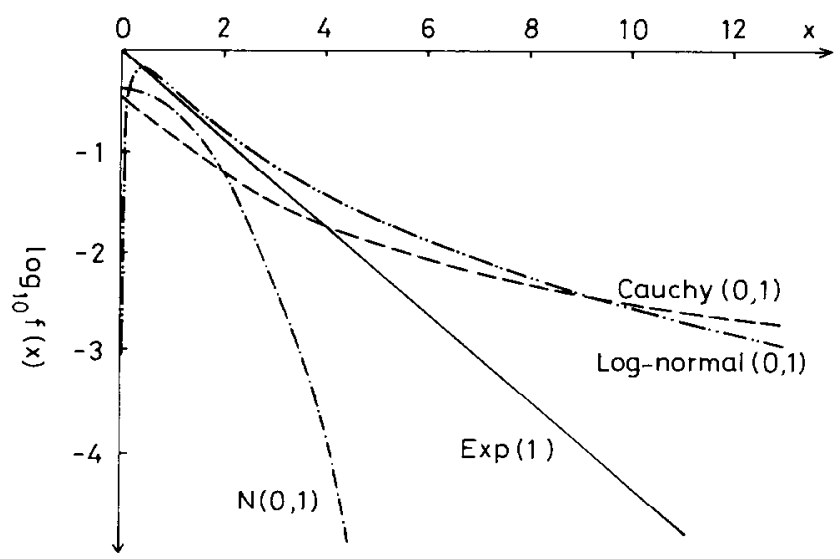

Fig. 2. Tail behaviors of several distributions. 
Table 1. It is interesting to notice that the tail behavior of the c.d.f. $F(x)$ reflects the limiting behavior of the constant $b_{n}$. In Figure 2 the density functions in log scale of the normal, exponential, lognormal and Cauchy distributions are plotted. They can be categorized into light-, medium- and heavy-tailed distributions according as the term $\lim _{n, \infty}\left[\left(-\log _{10} f(x)\right) / x\right]$ is infinite, a nonzero constant or zero, respectively.

For light-tail distributions such as normal distributions, the 'standard deviations' $b_{n}$ of extreme order statistics tends to zero. Therefore, $T_{r}$ are ideal test statistics asymptotically, i.e., the large sample power converges to one. However, the rate of convergence is much slower for normal distributions as compared with uniform distributions since the constant $b_{n}=1 / \sqrt{2 \log n}$ for normal $(0,1)$ distributions tends to zero more slowly as compared with the constant $b_{n}=1 / n$ for uniform $(0,1)$ distributions.

For medium-tail distributions such as exponential distributions, $b_{n}$ tends to a nonzero constant. Thus, the limiting power is some value strictly between the significance level and one. However, for heavy-tail distributions such as lognormal or Cauchy distributions, $b_{n}$ tends to infinity as $n$ tends to infinity. The power of $T_{r}$ decreases as $n$ increases and finally becomes $\alpha$ in the limiting case as $N \rightarrow \infty$ and $n / N \rightarrow \lambda$. In other words, the test statistics $T_{r}$ are worse for larger sample sizes and finally become useless as $n$ tends to infinity.

\section{A Monte Carlo simulation}

In this section we report the results of a Monte Carlo simulation which shows that the large sample results derived in Sections 3 and 4 are predictive of the actual behavior in small and moderate sample sizes. We simulated Rosenbaum's statistic $\left(T_{1}\right)$ with $k=3$ populations and significance level $=0.1$ for sample sizes $n=5,10$, 25,50 and 100 and under a wide variety of distributions: uniform $(0,1)$, normal $(0,1)$, exponential $(1)$, lognormal $(-0.5,1)$, and Cauchy $(0,1)$. For each of the distributions we considered the alternative where the $k$-th population is shifted to the right by one unit except for the uniform, which was shifted by 0.3 . We also considered a Lehmann alternative with $m=0.368$ (results independent of the underlying distribution). The results are shown in Figure 3. As predicted by the large sample results, the power increases towards one for the uniform and normal distributions, remains largely unchanged for the exponential and Lehmann alternatives and decreases for the lognormal and Cauchy distributions. Perhaps most dramatic is the sharp decrease in power for the long-tailed distributions, even for relatively small changes in sample size, such as from 5 to 10. Other (unreported) simulations for other statistics $\left(T_{r}\right)$ and significance levels showed similar results. We chose to report the results for $T_{1}$ since it is one of the most commonly used of this class of test statistics. 


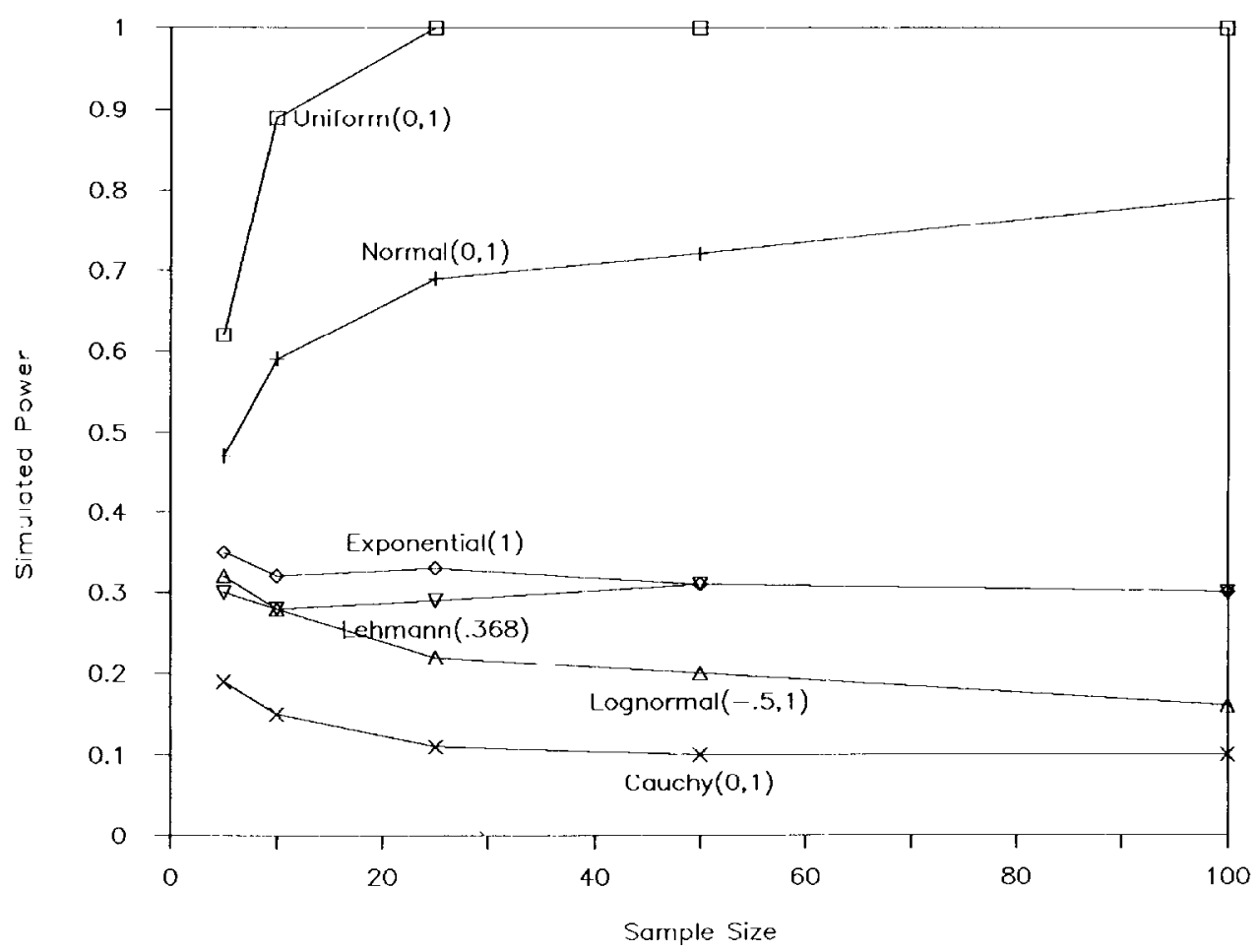

Fig. 3. Simulated power of $T_{1}$ for $k=3$ populations and significance level 0.1 . Each point represents 1000 replications.

These results naturally raise the question as to the optimal choice of $r$. Limited Monte Carlo simulations (not reported here) indicate that for long-tailed distributions, values of $r$ near $\frac{1}{2} N$ perform best, while for short-tailed distributions, values of $r$ that are small perform best.

\section{Relation to slippage tests}

We next indicate how the results described in this article apply to nonparametric slippage tests. The easiest case to analyze is Mosteller's test (Mosteller, 1948). The null hypothesis for the slippage test is again given by (2.1) but the alternative is simply that an unspecified population has slipped to the right of the others. This alternative hypothesis is quite different from the hypothesis considered in Section 2 and in some cases may be more practical. Thus this test would be especially appropriate for testing homogeneity where only one population is different from the others. This is a case that would be difficult for a test of homogeneity like the one-way anova F-test to detect. Mosteller selects as the population which is suspected to have slipped to the right as the one with the largest observation. The test statistic is then the 
number of observations in the suspected population larger than all the observations in the remaining populations. We will denote this test statistic $T_{1}^{*}$.

First, we will argue that the probability of a correct decision for the slippage test is less than the test proposed in Section 2 (which 'knows' which population is largest if any of them are). It is clear that $T_{1}^{*} \geq T_{1}$, since if the $k$-th population (assumed without loss of generality to be the one with the largest population mean) has the largest observation, then $T_{1}^{*}=T_{1}$, otherwise $T_{1}=0$. Thus the critical values for $T_{1}^{*}$ must be larger than the ones derived for $T_{1}$. Since a correct decision as to the slipped population is only made in the case when the $k$-th population gives the largest observations and since, for that case, $T_{1}^{*}=T_{1}$, the power of the test in Section 2 is larger than the probability of a correct decision using Mosteller's test. Thus, for heavytailed distributions like the lognormal, the probability of a correct decision will decrease to the significance level as the sample size increases.

Second, we argue that if the power of the test converges to one, then the probability of a correct decision in Mosteller's test also converges to one. If the power of the test based on $T_{1}$ converges to one then the largest observation comes the $k$-th population with probability approaching one. Thus $T_{1}^{*}=T_{1}$ with probability approaching one. Investigation of equation (4.6) shows that the critical value, $t$, has no bearing on the limiting result if $b_{n}$ converges to zero (the light-tailed case). Thus, $T_{1}^{*}$ and $T_{1}$ will behave similarly in the limit and both have limiting probability one.

The above discussions show that the nonparametric slippage tests will exhibit the same qualitative behavior as the location test. That is, they will have limiting probability of correct decision equal to one in some cases and equal to the significance level in other cases. This will be true even with distributions which, in practice, can look very similar, like the normal and lognormal distributions.

\section{Conclusions and discussions}

By generalizing Mosteller's and Rosenbaum's methods, nonparametric test statistics, $T_{r}$, based on the $r$-th maximum, are considered in this paper. We use $T_{r}$ as a typical example of a statistic used for nonparametric tests. The null distribution of $T_{r}$ has been derived analytically and it tends to a negative binomial distribution for large sample sizes. Two kinds of alternative hypotheses, Lehmann alternatives and pure-shift alternatives, are included to investigate the power of $T_{r}$. The concentration in this paper is the analytical analysis of large sample power which is simple and illustrative.

Under Lehmann alternatives, the large sample power of $T_{r}$ is independent of the population distributions but depends greatly on the order of the test statistic, $r$. Though the test $T_{r}$ with $r=1$ is very quick and simple in operation, its power is rather small and can be improved by a choice of larger $r$.

Under pure-shift alternatives, the large sample power of $T_{r}$ depends greatly on 
the tail behavior of the population distribution. For distributions which have finite upper range (no tails) such as uniform distributions, the large sample power tends to one no matter how small the shift is. For light-tail distributions such as normal distributions, the large sample power also tends to one but at a much slower rate. However, for medium-tail distributions such as exponential distributions, the increase in sample size does not contribute much in improving the power. The worst case is with heavy-tail distributions such as lognormal and Cauchy distributions, for which increasing the sample size only decreases the power and ultimately makes the test based on $T_{r}$ useless.

Many nonparametric mean-slippage tests, such as those proposed by Mosteller (1948), Conover $(1965,1968)$, Joshi and Sathe (1978), and others, are based on the comparison of maximum or minimum among the populations. It is reasonable to infer that they will exhibit properties similar to $T_{1}^{*}$. From the limiting behaviors of $T_{r}$, it is concluded that one should examine their data a little bit before jumping into nonparametric methods. Though the nonparametric test statistics are distribution-free under the null hypothesis, their power depends tremendously on the alternative and the population distributions. To sum up, without any idea of the population distributions, these nonparametric methods can be as unreliable as, or sometimes less reliable than, parametric methods.

Also, it should be noted that, in the limiting case, the proposed test $T_{r}$ with any fixed $r$ discriminates the pure-shift populations based only on the tail portion of the distributions. This probably is the reason why the test is satisfactory for no- or lighttailed distributions, bul nol for medium- or heavy-tailed distributions. This argument also suggests that $T_{r}$ with different ratios of order $r$ to sample size $n$ may emphasize different portions of the distributions for testing pure-shift alternatives and should perform better in practice.

\section{References}

Barnett, V. and T. Lewis (1984). Outliers in Statistical Data. Wiley, New York.

Bofinger, V.J. (1965). The $k$-sample slippage problem. Australian J. Statist. 7, 20-31.

Conover, W.J. (1965). Several k-sample Kolmogorov-Smirnov tests. Ann. Math. Statist. 36, $1019-1026$.

Conover, W. J. (1968). Two k-sample slippage tests. J. Amer. Statist. Assoc. 63, 614-676.

Folland, G.B. (1984). Real Analysis. Wiley, New York.

Galambos, J. (1978). The Asymptotic Theory of Extreme Order Statistics. Wiley, New York.

Joshi, S. and Y.S. Sathe (1978). A generalization of Mosteller's test. Comm. Statist. -Theory Methods 7, 709-715.

Joshi, S. and Y.S. Sathe (1981). A $k$-sample slippage test for location parameters, J. Statist. Plann. Inference 5, 93-98.

Lehmann, E.L. (1953). The power of rank tests. Ann. Math. Statist. 24, 23-43.

Mood, A.M., F.A. Graybill and D.C. Boes (1974). Introduction to the Theory of Statistics. McGrawHill, New York, 3rd ed.

Mosteller, F. (1948). A $k$-sample slippage test for an extreme population. Ann. Math. Statist. 19, 58-65. Mosteller, F. and J.W. Tukey (1950). Significance levels for a $k$-sample slippage test. Ann. Math. Statist.

21, 120-123. 
Neave, H.R. (1966). A development of Tukey's quick test of location. J. Amer. Statist. Assoc. 61, 949-964.

Neave, H.R. (1972). Some quick tests for slippage. The Statistician 21, 197208.

Neave, H.R. (1973). A power study of some tests for slippage. The Statistician 22, 269-280.

Neave, H.R. (1975). A quick and simple technique for general slippage problems. J. Amer. Statist. Assac. 70, 721-716.

Neave, H.R. and C.W. Granger (1968). A Monte Carlo study comparing various two-sample tests for differences in means. Technometrics 10, 509-522.

Rosenhaum, S. (1954). Tables for a nonparametric test of location. Ann. Math. Statist. 25, 146-150.

Rosenbaum, S. (1965). On some two-sample nonparametric tests. J. Amer. Statist. Assoc. 60, $1118-1126$.

Tukey, J.W. (1959). A quick, compact, two-sample test to Duckworth's specifications. Technometrics 1, 31-48. 